\title{
Liste verwendeter Symbole
}

$\neg \mathrm{p} \quad$ nicht-p, d. h. Negation der Proposition $\mathrm{p}$

$S \rightarrow T \quad$ aus $S$ folgt logisch $T$

$\mathrm{S} \nrightarrow \mathrm{T} \quad$ aus $\mathrm{S}$ folgt nicht logisch $\mathrm{T}$

$\mathrm{T} \nleftarrow \mathrm{S} \quad$ aus $\mathrm{S}$ folgt nicht logisch $\mathrm{T}$

$\mathrm{S} \Leftrightarrow \mathrm{T} \quad \mathrm{S}$ und $\mathrm{T}$ sind logisch äquivalent

$A \cap B \quad$ Schnittmenge aus $A$ und $B$, die Menge, die nur aus den $A$ und $B$ gemeinsamen Elementen besteht.

$A \cup B \quad$ Vereinigungsmenge aus $A$ und $B$, die Menge, die alle Elemente aus $A$ sowohl wie $B$ umfasst.

$\{\mathrm{x} \mid \mathrm{x}$ ist rot $\} \quad$ Mengendarstellung durch Beschreibung, hier: die Menge all der Dinge, die rot sind.

$A \neq B$

A und $B$ sind nicht gleich

$\emptyset$

leere Menge

A\B

$A \subseteq B$

Menge A minus der Elemente, die auch in $B$ sind

$a \in B$

Menge $A$ ist Teilmenge von Menge $B$

$\mathrm{a}$ ist Element von Menge $\mathrm{B}$

【S】

$\langle\pi \bullet \varepsilon>$

$\lambda$ p. $\langle p \bullet \varepsilon>$ wörtliche Bedeutung des Satzes S

zweidimensionale Bedeutung: propositionale Bedeutung $\pi$ und expressive Bedeutung $\varepsilon$

Operator, der der Proposition p einen expressiven Inhalt $\varepsilon$ hinzufügt

Steigakzent

Fallakzent 
